\title{
Drug Delivery and Distribution System of Ebola in Sierra Leone Xiangjie Xie ${ }^{1, a}$ \\ ${ }^{1}$ North China Electric Power University, Baoding, 071000, China \\ a2460388161@qq.com
}

Keywords: Ebola,drug manufacturing function,drug delivery,Differential Equations and Iteration

\begin{abstract}
In 2014, Ebola, which wreak havoc West Africa was alarmingly spread in The Republic of Guinea, Sierra Leone and Liberia.But the world medical association has announced that their new medication could stop Ebola and cure patients whose disease is not advanced. This paper confirms the distribution method. Firstly, seting up an exponential function to describe the speed of manufacturing of the drug, distributing the drugs depends on the rate of infected people. According to the model , the number of the next day's patients can be calculated. We can get result that 33 days after the control, the Ebola is eradicated by iterating data.
\end{abstract}

\section{Introduction}

Before building the model,we get Propagation of Ebola and classify the people into three different parts. With the using of SIR model,the convert relationships are shown clearly.Then we deal with the evaluation function through AHP. Distance, population, cum-patients, the increase rate of patients are four factors that considering. To get the shortest path.

In this paper, we analyze the production of drugs, the possible feasible delivery systems and the quantity of the medicine needed.

\section{Assumption}

(1) In order to simplify the model, the treatment cycle is not to be considered. In otherwords, a dose of the drug could have a good effect and remove the patient to R set.

(2) The cycle of the production is one day.

(3) The pharmaceutical factory is controlled by the decision maker within the scope of bearing .In other words , any number of the medicines can be gotten if it is less than a max number.

(4) Adopting a 40×14 0- 1 matrix to represent the ith day and distribute the jth district drugs. We initial all values in matrix as 0 .

(5) The susceptible number $S$ and infectious number I are confirmed that they are the initial solutions of the program iteration.

(6) The regional distribution of drugs, the urban population $\mathrm{N}$, the rateof the susceptible people $\mathrm{S}$ and infectious patients $\mathrm{I}$, the number of susceptible Sand infectious patients have a close relationship .We will distribute more medicines to the district which has a bigger value. So a formula to represent the medicines needed quantitatively can be assigned.

The capacity of the production is limited by the technology, material andother factors and approximately obey the function $f(t)=2 \times e^{\sqrt{t / 2}}$.

With the growth of the time, the proportion of the medicine whichpharmaceutical factories product and total number of cases is rising. So the function about the proportion is $g(t)=0: 01 \times t$. 


\section{The Model Calculation and Results}

\subsection{Calculation}

If the generative medicine amount in kth day is less than the number of the patients ,the medicines distributed throughout the country obey the function $\mathrm{f}(\mathrm{k})$. Otherwise, the number of medicines is NI. Express by the formula:

$$
\left\{\begin{array}{l}
\text { medicine }=f(k), \leq f(k) N i \\
\text { medicine }=N i, f(k)>N i
\end{array}\right.
$$

According to the formula $\mathrm{l}(\mathrm{t})=0: 01 \mathrm{t}$ which we set to represent the proportion of thepersons who are cured by medicines and all patients, then proceed to screen.

(1) $\operatorname{rate}(\mathrm{t})=$ medicine $(\mathrm{t}) / \mathrm{Ni}(\mathrm{t})$

(2) If rate $(\mathrm{t})<\mathrm{g}(\mathrm{k})$, getting the minimum city by comparing test and remove it.

(3) Repeat the above step , continuing screening the other cities till the rate $(t) \geq g(k)$.

According to the proportion of the each city's patients and the national patients, then distribute medicines to each city. Express by the formula:

$$
\text { medicine }_{k}=\frac{\text { medicine }_{t}}{\sum I(t)}
$$

According to the improvement of the SIR Model, we get following fomulas:

$$
\left\{\begin{array}{l}
s_{t}=\frac{1+\lambda s_{t-1}+\lambda I_{t-1}-\lambda m_{t}-\sqrt{\left(1+\lambda s_{t-1}+\lambda I_{t-1}-\lambda m_{t}\right)^{2}-4 \lambda s_{t-1}}}{2 \lambda} \\
I_{t}=-m_{t}-s_{t}+s_{t-1}+I_{t-1}
\end{array}\right.
$$

Finally, getting matrixs about $\mathrm{I}$ and $\mathrm{X}$ and find that $\mathrm{I}$ is convergence and become 0 after 33 days . That represent Ebola is eradicated. Ebola is controlled and patients I become 0. The sum drugs for each day and each capital is shown in figure 1.
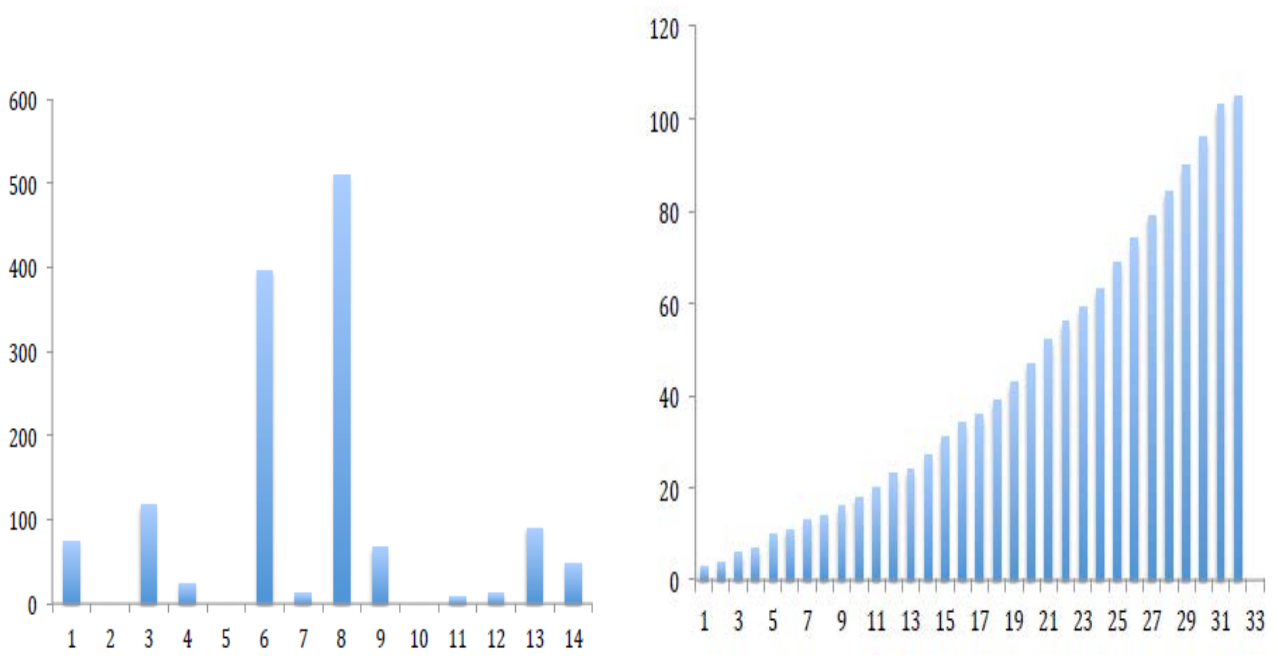

\subsection{Results}

Figure 1.The sum drugs for each day and each capital

(1) The trend of spread of Ebola is gained,the results is as figure 2 .

(2) The quantity of the medicin needed and it's 1315.

(3) The possible and feasible delivery systems are developed.

(4) The location of delivery is Kenema. 
(5) The speed of manufacturing of the medicine is clear.

(6) Besides different point of time that the epidemic start to be controlled have different influence on the epidemic situation, which is showed in figure 2.

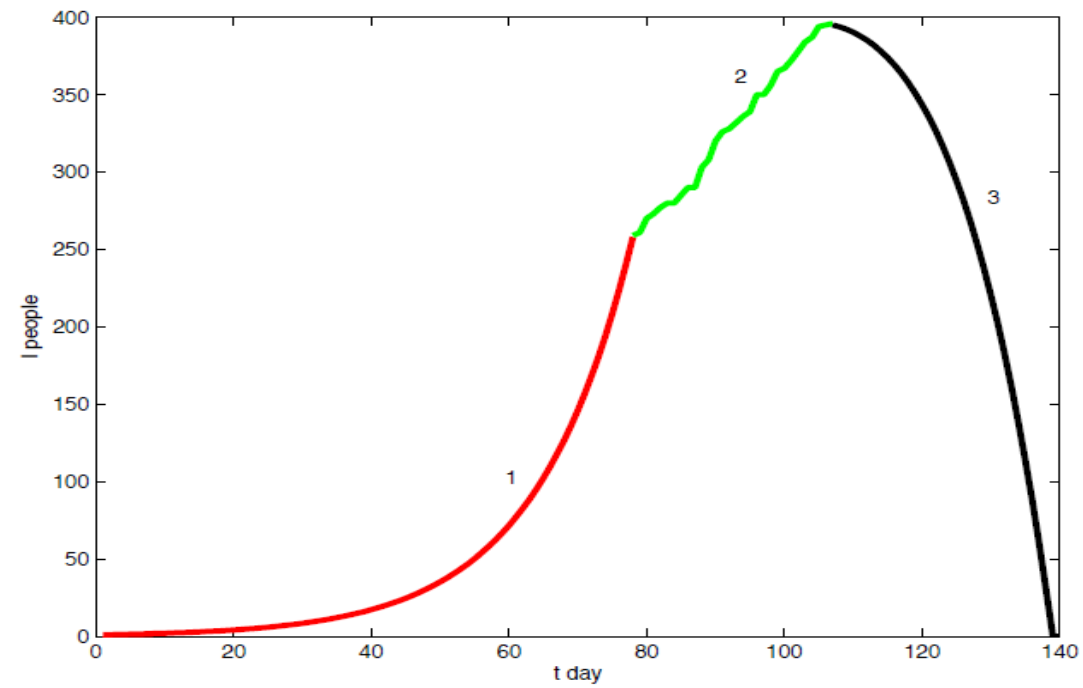

Figure 2.The trend of whole period

\section{Conclusion}

\subsection{Sensitivity Analysis}

According to the analysis of the medicine delivery,speed of manufacturing of the drug would influence the spread of Ebola,thus our final result would also be affected. In reality,it's necessary for us to determine the speed of manufacturing of the drug, so that we can decide how many doses of the medicine should be manufactured a day. Thus sensitivity analysis of the manufacture speed can be maked.

To solve the model,we define the function of manufacture speed as $f(t)=2 e^{\sqrt{t / 2}}$. Then we change it into $f(t)=2 e^{\sqrt{t / 3}}$ and $f(t)=2 e^{\sqrt{t}}$ to look over how the speed influence the final result.We take Kenema for example to make the curve of the changing total number of patients.From the figure above,the sensitivity of the number of susceptible ndividuals is connected with the speed of manufacturing of the drug. The faster the speed is,the more Ebola will be controlledand the shorter the period of the epidemic situation will be.

The sensitivity of the number of susceptible individuals is also related to the time $t$,mesured in days.It would decrease with the increase of the speed.In the beginning,it decrease so slowly that increase the speed is of no use.But when the number of patients become small,increasing the speed could observably control the epidemic. 


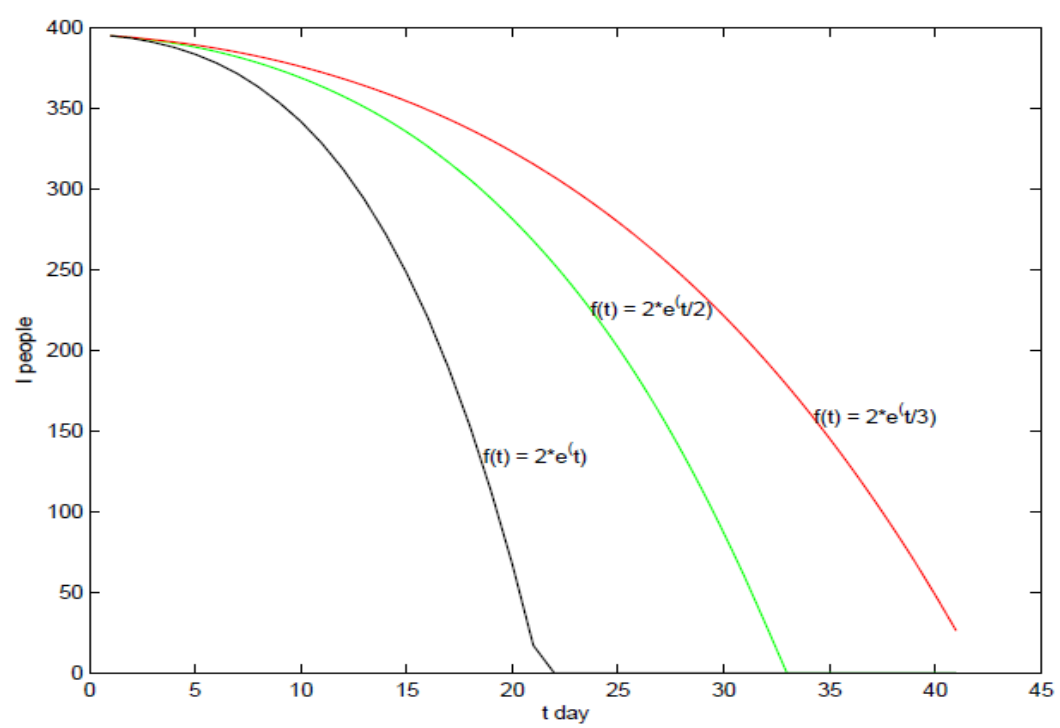

Figrure 3.The Sensitivity Analysis

\subsection{Strengths and weakness}

\subsubsection{Strengths}

(1) Our model are realistic, sensible, and useful to solve the problem.

(2) Choosing a Representative country Sierra Leone,which not only simplify the problem,but also can be extended to eradicate Ebola all around the world.

(3) We use the difference method to solve the differential equation model ,resulting in a realistic and reasonable consequence.

(4) Except for the factors that are indicated in the topic,different point of time that the epidemic start to be controlled have different influence on the epidemic situation.

\subsubsection{Weakness}

(1) Ignoring gender and age of the people,which will cause little erros in our analysis.

(2) Only delivering the drugs by the rate of the patients,which is not a pefect model.

(3) Not considering the effecting time of the drug and ignore the vaccine of Ebola.

(4) Assuming a function to describe the quantity of the medicine at each time of t,which lacks of the scientific basis.

\section{References}

[1]. Halloran ME, Ferguson NM, Eubank S, et al. Modeling targeted layered containment of an infl uenza pandemic in the United States. Proc Natl Acad Sci USA 2008; 105: 4639-44.

[2]. Zhang Zuowen.Wang Changen.Feng Xuelian.2003.The epidemiology of SARS.

[3]. Wang Nengchao.1984.The concise course of numerical analysis.

[4]. Pandey A, Atkins KE, Medlock J, et al. Strategies for containing Ebola in west Africa. Science 2014; 346: 991 - 95.

[5]. Merler S, Ajelli M, Fumanelli L, Vespignani A. Containing the accidental laboratory escape of potential pandemic infl uenza viruses. BMC Med 2013; 11: 252. 